\title{
Composition of Neurospora crassa Mycelium Color
}

\author{
Jaruwan Chutrtong ${ }^{1 *}$, Waradoon Chutrtong ${ }^{2}$, Narumon Boonman ${ }^{3}$ \\ ${ }^{1}$ Industrial Microbiology Department, Faculty of Science and Technology, Suan Sunandha Rajabhat \\ University, Bangkok, CO 10300 Thailand. \\ ${ }^{2}$ Chemistry Department, Faculty of Science, Srinakharinwirot University, Bangkok, CO 10110 Thailand. \\ ${ }^{3}$ Industrial Microbiology Department, Faculty of Science and Technology, Suan Sunandha Rajabhat \\ University, Bangkok, CO 10300 Thailand.
}

* Corresponding author. Tel.: 6614187988; email: jaruwan.ch@ssru.ac.th

Manuscript submitted November 10, 2017; accepted May 12, 2018.

doi: 10.17706/ijbbb.2018.8.4.237-244

\begin{abstract}
Microbial products have been used by human for a long time. One of the products is color. Researches were done to find more products that can be useful. Therefore, this research studied about color extracted from mycelium of Neurospora crassa, fungus in phylum Ascomycota, which has not been used before. Mycelium was extracted with absolute ethanol. The color was orange. The optimum wavelength for the colorimetric measurement was $420.5 \mathrm{~nm}$ and the optical density (O.D.) or absorbance of the color extract was 0.222 . From the result of experiment, it found that the appropriate solution to separate the colorants extracted to pure substance for studying the composition is acetone and chloroform. After separated with TLC aluminum sheet, there are five groups of separable substances.
\end{abstract}

Key words: Color, composition, mycelium, Neurospora.

\section{Introduction}

Color is an important factor that helps consumers to attract the product. The colors used in the manufacture of various products are both synthetic and natural colors. At present consumers focus on buying natural ingredients. This is due to the realization of the danger of synthetic substances that will affect the health in the long- run. Therefore, the use of natural colors in the product will be part of the promotion of that product. The color of the microorganism is one of natural color. The use of microorganisms in pigment production has the advantage of producing faster and more efficiently than the production of pigments from plants and animals. Genetic improvement of microorganisms to make the color more or better quality is easy. Because use less production space, it can increase or decrease the production size easily. In addition, the colorants obtained from microorganisms are not toxic to the environment [1]. If study color production from microbial on variety of colors, it will be an option to develop products that are acceptable to consumers in terms of product safety to consumers and environment. It may also be possible to develop the process to the industrial level. It will be economic development for the country.

Microorganisms have been used for a long time for production of pigments and use in many industries such as fish industry already, for example to enhance the pink color of farmed salmon. The most well known color produce fungus is Monascus [2]. Neurospora crassa is a type of red bread mold of the phylum Ascomycota. The genus name, meaning "nerve spore" in Greek [3], refers to the characteristic striations on 
the spores. $N$. crassa lives mainly in tropical and sub-tropical regions. It can be found growing on dead plant matter after fires. N. crassa is used as a model organism because of its easy life cycle [4], [5]. Neurospora was used by Edward Tatum and George Wells Beadle in their experiments for which they won the Nobel Prize in Physiology or Medicine in 1958 [6], [7] and many scientists also worked on Neurospora [8].

The objective of this research is to investigate the method of extraction color from fungus, Neurospora crassa, and to study its properties. The results of research will use to evaluate the use of color from this fungus.

\section{Procedure}

\subsection{Microorganism and Culture Conditions}

The microorganisms used in this study were Neurospora crassa. The stationary cultures were performed in $500-\mathrm{cm}^{3}$ Erlenmeyer flasks containing $250 \mathrm{~cm}^{3}$ of potato dextrose agar. Make slope surface to increase the surface area. The cultures were incubated for 21 days at $28^{\circ} \mathrm{C}$ [9].

\subsection{Color Extraction from Mycelium}

The conventional extraction technique was used to carry out the extraction of color from Neurospora crassa mycelium. This method based on the use of organic solvents being solid-liquid extraction (SLE) [10] [11], the most widely used for solid samples [12]. Mycelium were collected to other flask, homogenized and extracted with absolute ethanol, added only cover the mycelium. Leaved until the mycelium color come out then filter the color extract out with filter paper (whatman no.1).

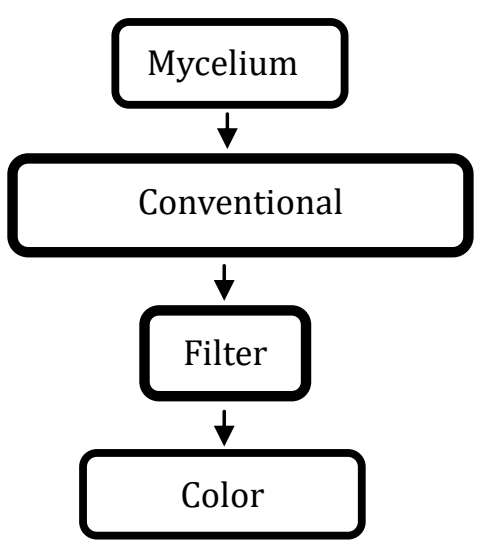

\subsection{Study Concentration of Color Extract}

By measuring with spectrophotometer, UV-VIS Spectrophotometer (PerkinElmer, Lambda 25) [13] [14], the concentration of solutions was found. The wavelength at which absorbance was greatest needs to be determined. The process recorded the absorbance over the range of $350 \mathrm{~nm}$ to $650 \mathrm{~nm}$, usually in intervals of $25 \mathrm{~nm}$. When got the optimum wavelength, record the value of the optical density (OD) of the substance.

\subsection{Study on Color Separation Conditions}

Because the composition of the extract substance was not known, so it must study what were the components. The study was conducted using TLC to see how many substances they contained. First, removed absolute ethanol from the extract by evaporating until dry. Next, dissolved the remain stain with little amount of methanol : chloroform (1:1). Then plot solvent on 25 TLC aluminum sheet silica gel 60 F254 (Merck). After that developed aluminum sheet in vary systems as follows to find the suitable condition for separate the components.

- dichloromethane: hexane $=100: 0,80: 20,20: 80$ and $2: 98$ 
- acetone : hexane $=10: 90,50: 50,100: 0$

- acetone : chloroform $=5: 95,8: 92,10: 90,15: 85,20: 80,25: 75,30: 70,50: 50,0: 100$

- methanol : water $=100: 0,70: 30,50: 50,25: 75$ and $0: 100$

- methanol : hydrochloric $=99: 1$

- methanol: Ammonia = $99: 1$

\subsection{Composition of the Extract}

The suitable solvent from result of the experiment in 2.4 was used as mobile phase in column chromatography for separate composition of the extract.

\section{Result}

\subsection{Color Extraction from Mycelium}

After flooding mycelium with absolute ethanol, leaved it until the color dissolved. The extract was liquid with dark orange color.

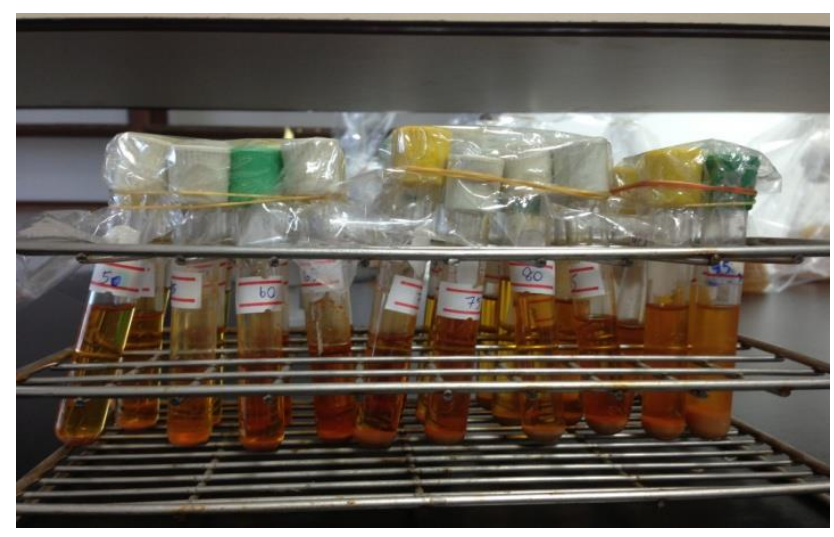

Fig. 1. Mycelium extract.

\subsection{Concentration of Color Extract}

The optimum wavelength for measure the mycelium extract was at $420.5 \mathrm{~nm}$. The absorbance of extracted color is 0.222 .

\subsection{Color Separation Conditions}

The experiment was conducted to determine the optimum conditions for the separation of the extract components. The result showed as followed.

1) Character of extract in dichloromethane and hexane system as in Fig. 2

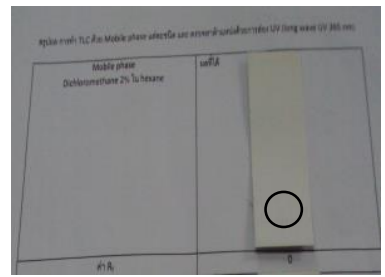

a

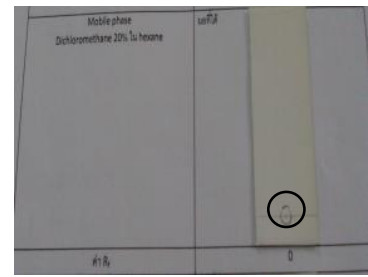

b

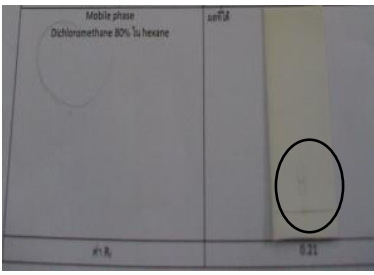

C

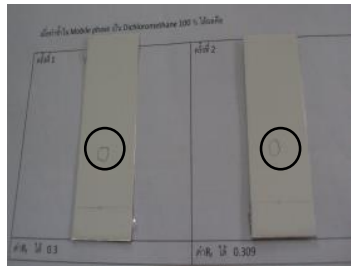

d

Fig. 2. Separation of substances in dichloromethane and hexane system at ratio = 2: 98(A), 20: 80(B), 80: 20(C) and 100: 0(D). 
2) Character of extract in acetone and hexane system as in Fig. 3

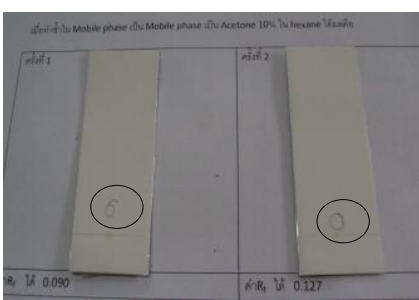

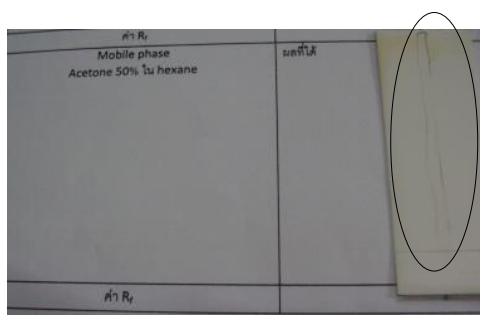

h

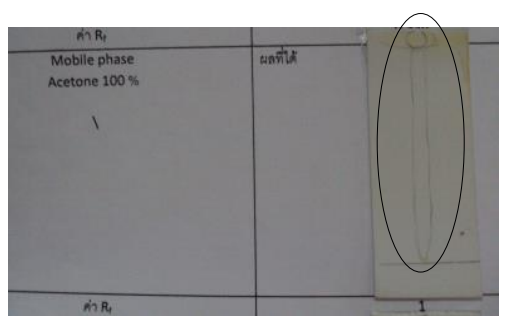

Fig. 3. Separation of substances in acetone and hexane system at ratio $=10: 90(A), 50: 50(B)$ and 100: $0(C)$.

3) Character of extract in acetone and chloroform system as in Fig. 4, 5 And 6

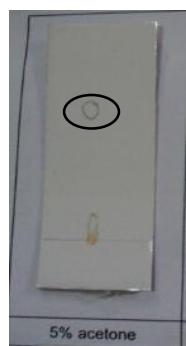

a

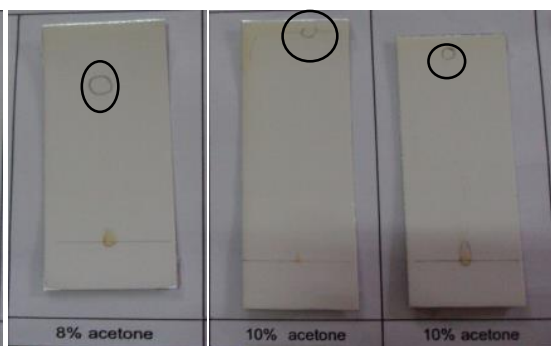

d

Fig. 4. Separation of substances in acetone and chloroform system at ratio $=5: 95(A), 8: 92(B)$ and 10: $90(\mathrm{C}, \mathrm{D})$.

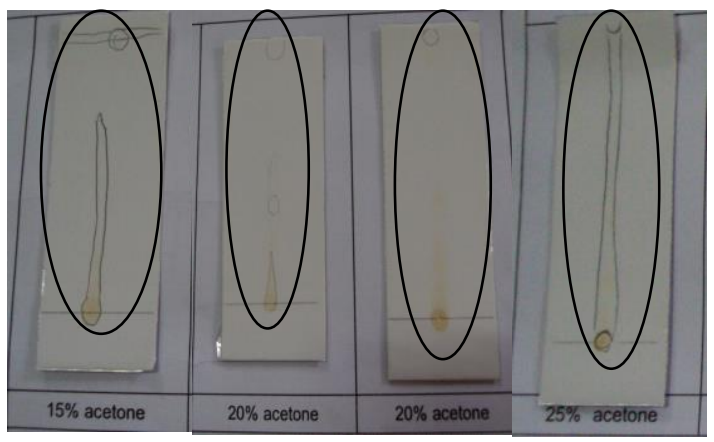

a

b

C

d

Fig. 5. Separation of substances in acetone and chloroform system at ratio $=15: 85(A), 20: 82(B, C)$ and 25: 75(D).

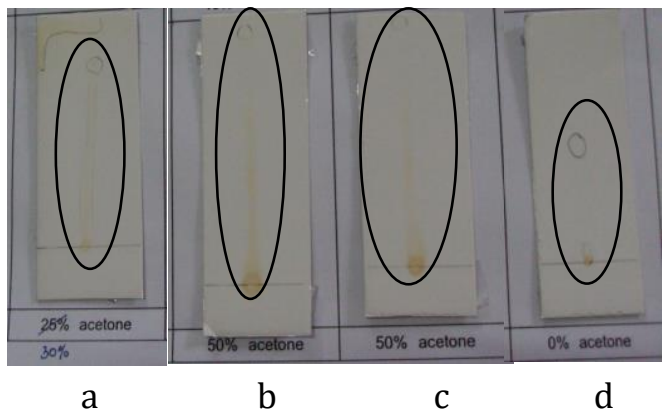

Fig. 6. Separation of substances in acetone and chloroform system at ratio $=30: 70(A), 50: 50(B, C)$ and 0 : $100(D)$. 
4) Character of extract in methanol and water system as in Fig. 7

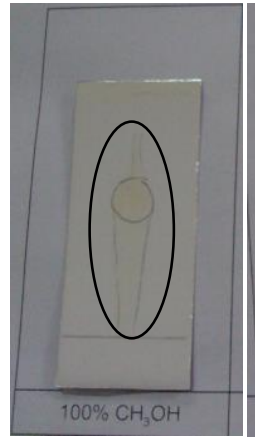

a

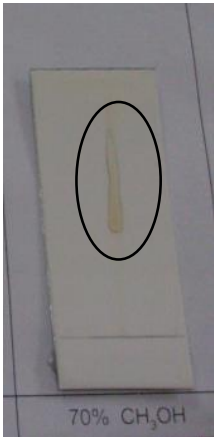

$\mathrm{b}$

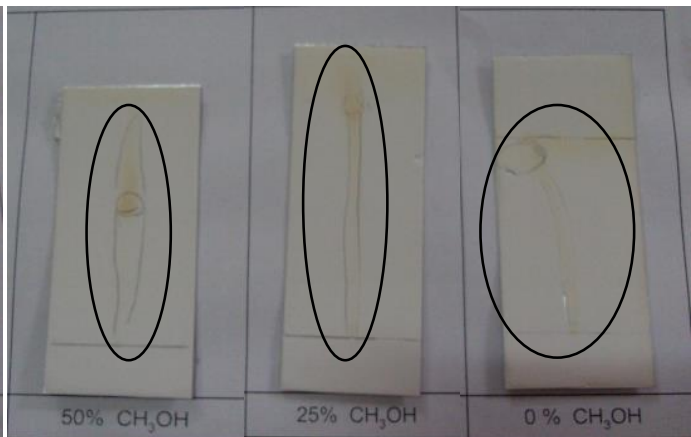

d

e

Fig. 7. Separation of substances in methanol and water system at ratio = 100:0(A), 70:30(B), 50: 50(C), 25: 75(D) and 0: 100(E)

5) Character of extract in methanol system (with hydrochloric acid) as in Fig. 8.

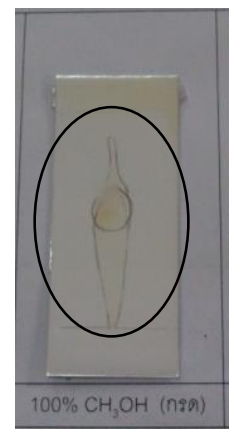

Fig. 8. Separation of substances in $100 \%$ methanol, acid condition.

6) 3.3.6 Character of extract in methanol (with ammonia) as in Fig. 9.

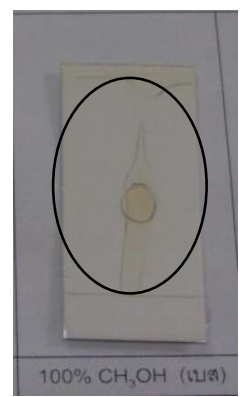

Fig. 9. Separation of substances in $100 \%$ methanol, base condition.

\subsection{Composition of the Extract}

The result of separation using column chromatography found that there were five groups of separable substances. The first one was yellow substance which separated when used acetone: chloroform at ratio 5: 95. The second one was orange substance which separate when used acetone: chloroform at ratio 8: 92. The third one was yellow substance which separate when used acetone: chloroform at ratio 50: 50. And the last one was yellow substance which separate when use water. Moreover, there was brown substance which could not separate from the column, adhered to the silica gel. 


\section{Conclusion}

\subsection{Mycelium Color Extraction with Ethanol}

The color of Neurospora crassa mycelium extracted with ethanol is orange, same as visible color. Measured with spectrophotometer at $420.5 \mathrm{~nm}$ (the best wavelength of the extract), OD value was 0.222. The intensity of this color depends on the amount of the extracted solution. If using a very large amount of solvent, the color is diluted. Although the volume of solvent is constant but the amount of mycelium can't put exactly. It is difficult to have the same quantity extract.

\subsection{Color Separation Conditions}

The results of using dichloromethane and hexane as solution to separate the extracted components, it showed that hexane is not suitable solvent. Sample did not move to top of TLC sheet. When increased the ratio of dichloromethane, sample moved faster. When replaced the solution with acetone and hexane, it was found that acetone can partially remove the sample. There are some non-moving parts.

The mixture of solution was changed to be acetone and chloroform. The mobility and separation of the substance was better. After that higher polarity solution was used, the mixture of methanol and water. Its ability of moving and separation sample was better than acetone and chloroform. Based on the isolation study, it could conclude that the appropriate solution for separating the colorants extracted from Neurospora crassa mycelium was acetone and chloroform.

\subsection{Composition of the Extract}

Separated with acetone and chloroform in Chromatography column, the first extractable substance is yellow. After the first substance separate, increase the ratio of acetone to make the second substance, which is orange, separate. Then increase the ratio of acetone up to the ratio at 50: 50 to make the third substance, which is a yellow substance, separate. To remove remaining substance from column, the higher polarity solvent, water, is used. Water made the fourth substance separate. It still had the remaining attached to the silica gel at the top of the column. It could conclude that the extracted material has at least 5 mixtures that used acetone, chloroform and water separated 4 types. The rest cannot be separated with solution.

The results of these experiments can use to separate these compounds (color extract from Neurospora mycelium) in order to study the components in detail with more efficient instruments, such as Infrared Spectroscopy (IR)and Nuclear magnetic resonance spectroscopy (NMR), in order to know the structure of the substance. Structure of the substance let us know about the properties of the substance. This allows us to evaluate the benefits of this extract to be applied in future.

\section{Acknowledgment}

The author would like to thank Suan Sunandha Rajabhat University, Bangkok, Thailand for providing fund, necessary equipment and laboratory area.

\section{References}

[1] Casida, L. E. (2007). Industrial Microbiology, 2nd ed. New York: John Wiley and Sons, Inc.

[2] Broder, C. U., \& Koehler, P. E. (1980). Pigment produced by Monascus purpureus with regard to quality. J. Food Sci., 45, 579-583.

[3] Aramayo, R., \& Selker, E. U. (2017). Neurospora crassa, a model system for epigenetics research. Cold Spring Harb Perspect Biol., 5(10), 1-18.

[4] Davis, R. H., \& Perkins, D. D. (2002). Neurospora: A model of model microbes. Nature Rev. Genet., 3, 397-403. 
[5] Roche, C. M., Loros, J. J., Mccluskey, K., \& Glass, N. L. (2014). Neurospora crassa: Looking back and looking forward at model microbe. American Journal of Botany, 101(12), 2022-2035.

[6] Rowland, H. D., \& David, D. P. (2002). Neurospora: A model of model microbes-nature reviews. Genetics, 3, 7-13.

[7] Betina, V., Janstová, D., \& Spisiaková, J. (1979). Effects of antibiotics on the life cycle of Neurospora crassa. J. Folia Microbiologica., 20, 340-345.

[8] David, D. P., Alan, R., \& Matthew, S. S. (2001). The Neurospora compendium. Chromosomal Loci. (1st ed.). San Diego, Academic Press.

[9] Chutrtong, J. (2015). Comparison of corncob and corn using as fungal culture medium. Procedia-Social and Behavioral Sciences, 197, 797-800.

[10] Glass, N. L., \& Saupe,S. J. (2002). Vegetative incompatibility in filamentous ascomycetes. Molecular Biology of Fungal Development. New York: Marcel Dekker.

[11] Chew, A. L. C., Desjardin, D. E., Tan, Y. S., Musa, M. Y., \& Sabaratnam, V. (2015). Bioluminescent fungi from Peninsular Malaysia-A taxonomic and phylogenetic overview. Fungal Divers, 70, 149-187.

[12] Hi, X. L., Li, X. L., Lu, Y. P., \& He, Q. (2015). Composition and color stability of anthocyanin-based extract from purple sweet potato. Food Sci. Technol., 35(3), 2015.

[13] Kaskova, Z. M., et al. (2017). Mechanism and color modulation of fungal bioluminescence. Science Advances, 3(4), 1-8.

[14] Werawatganone, P., \& Muangsiri, W. (2011). Effect of micelles and ph on stability of clitoria ternatea color extract. J. of health research, 25(2), 55-60.

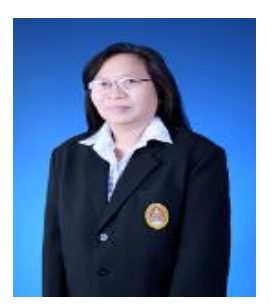

Jaruwan Chutrtong was born in Bangkok Thailand on January 3, 1965. She got the bachelor of science (B.Sc.) in biology (microbiology) from Srinakharinwirot university, Bangkok Thailand in 1985; the master degree in microbiology from Kasetsart university, Bangkok Thailand in 1994.

She worked as medical scientist in Regional Medical Sciences Center in Nakhon Ratchasima between 1985-1988. In 1988-1994, she worked as medical scientist in the Department of Medical Sciences, Ministry of Public Health in Bangkok. After that, from 1994 - now, she is lecturer in Industrial Microbiology Department, Faculty of Science and Technology Suan Sunandha Rajabhat University, Bangkok Thailand. Previous publications:

1. J. Chutrtong, "Comparison of Corncob and Corn Using as Fungal Culture Medium," Procedia-Social and Behavioral Sciences, vol.197, pp. 797-800, 2015.

2. J. Chutrtong, "Survival of Probiotic Bacteria in Freeze-Dry Yogurt Starter Cultures Storage at 4 and 30 Degree Celsius," Procedia-Social and Behavioral Sciences, vol.191, pp. 2219-2225, 2015.

3. J. Chutrtong, "Activity beyond classroom enhance understaning of science," Economic and Social Development: Book of Proceedings, pp. 412-415, 2016.

Asst. Prof. Jaruwan Chutrtong: memberships - committee of Educational Professional Foundation

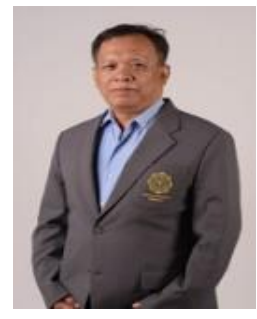

Waradoon Chutrtong was born Bangkok Thailand on 11 January 1959. He got the bachelor of science (B.Sc.) in chemistry from Ramkhamheng university, Bangkok Thailand in 1984; the master degree in chemistry (analytical) from Kasetsart university, Bangkok Thailand in 1995.

He worked as medical scientist in Regional Medical Sciences Center in Nakhon Ratchasima between 1986-1989. In 1989-1995, he worked as medical scientist in the 
Department of Medical Sciences, Ministry of Public Health in Bangkok. After that, from 1995 - now, he is lecturer in chemistry department, faculty of science, Srinakharinwirot university, Bangkok Thailand.

Asst. Prof. Waradoon Chutrtong: memberships - committee of Council of science and technology professionals.

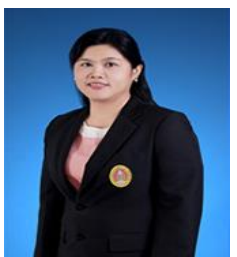

Narumon Boonman was born in Chuntaburi Thailand on26 April 1980. She got the bachelor degree in biology from Mahidol university, Bangkok Thailand in 2002; the master of science (M.Sc.) in microbiology from Mahidol university, Bangkok Thailand in 2007; the doctorate degree (Ph. D.) in microbiology from Mahidol university, Bangkok Thailand in 2012.

From 2012 - now, she is lecturer in industrial microbiology department, faculty of science and technology Suan Sunandha Rajabhat university, Bangkok Thailand. Previous publications:

1. N. Boonman, S. Wiyakrutta, N. Sriubolmas and A. D. Chusattayanond "Acanthamoebicidal activity of Fusarium sp. Tlau3, an endophytic fungus from Thunbergia laurifolia Lindl.,"Parasitology Research, Vol. 103, no.5, pp. 1083-1090, 2008.

2. N. Boonman, S. Prachya, A. Boonmee, P. Kittakoop, S. Wiyakrutta, N. Sriubolmas, S. Warit and A. D. Chusattayanond, "In vitro acanthamoebicidal activity of fusaric acid and dehydrofusaric acid from an endophytic fungus Fusarium sp. Tlau3.," Planta Med, vol. 78, no.14, pp.1562-7, 2012. 